\title{
Silencing of the Escherichia coli bgl operon by RpoS requires Crl
}

\author{
Karin Schnetz \\ Tel: +49221 4703815. Fax: +49221 4705975.e-mail: schnetz@uni-koeln.de
}

Institut für Genetik, Universität zu Köln, Weyertal 121, 50931 Köln, Germany

\begin{abstract}
Silencing of the Escherichia coli bgl operon is mediated by histone-like protein H-NS and affected by other pleiotropic regulators, including sigma factor RpoS. Silencing is relieved and the bgl operon is activated in hns mutants and by mutations that map in the vicinity of the bgl promoter. However, the expression level of activated bgl operon derivatives varies with the strain background. Here it is shown that the repression of the bgl operon by RpoS requires Crl. Crl is a protein that is necessary for the RpoS-dependent expression of the csgBA operon and that enhances the expression of other RpoS-dependent genes. In a Crl-negative strain RpoS had no effect on the bgl operon. The crl gene maps close to the proBA locus in the lac operon region and is deleted in many commonly used $E$. coli strains. Crl may therefore account for some of the observed strain-dependent variations of $\mathrm{bgl}$ operon expression levels and effects of pleiotropic regulators on bgl operon regulation.
\end{abstract}

Keywords: $\beta$-glucoside, pleiotropic regulation, H-NS, lac operon region

\section{INTRODUCTION}

The $b g l$ operon, encoding the gene products necessary for the fermentation of aryl- $\beta$-glucosides, is silent in wild-type Escherichia coli (Schaefler \& Maas, 1967). The abundant histone-like nucleoid-structuring protein H-NS is essential for $b g l$ silencing and in $h n s$-null strains the operon is expressed at high levels (Defez \& de Felice, 1981; Higgins et al., 1988). The activity of the CRP/ cAMP-dependent $b g l$ promoter is also enhanced and expression of the operon is activated by spontaneous mutations that disrupt a silencer sequence located upstream of the promoter, and by point mutations that improve the CRP-binding site (Reynolds et al., 1981, 1986; Schnetz \& Rak, 1992; Singh et al., 1995). Silencer sequences located downstream of the promoter contribute to silencing of the operon (Schnetz, 1995).

The expression level of the activated $b g l$ operon derivatives and the effect of mutations that partially disrupt the $h n s$ gene can vary with the strain background (Higgins et al., 1988; Lopilato \& Wright, 1990; Tsui et al., 1994; Free et al., 2001). In addition to H-NS and CRP, the $b g l$ operon is affected by other pleiotropic regulators including the DNA-bending protein FIS, which competes with CRP for binding (Caramel \& Schnetz, 2000), and the transcriptional-regulator-like proteins LeuO and $\mathrm{BglJ}$ (Giel et al., 1996; Ueguchi et al., 1998). RpoS, the key regulator in the stress response of E. coli and the H-NS homologue StpA are necessary for silencing of the wild-type $b g l$ operon by a truncated $\mathrm{H}$ NS protein lacking its C-terminal DNA-binding domain (Free et al., 1998, 2001; Ohta et al., 1999). Furthermore, RpoS downregulates the expression of activated $b g l$ operon alleles (Dole et al., 2002). Interestingly, the up to 50 -fold repression of the $b g l$ operon by RpoS is based on the amplification of a moderate (two- to threefold) repression of the transcription rate by RpoS via a second, post-transcriptional level of regulation involving the specific antiterminator protein BglG. BglG is encoded by the first gene of the operon. At low transcription rates BglG is limiting and transcription halts at rho-independent transcriptional terminators $t 1$ in the leader and $t 2$ within the operon. If the transcription rate increases above a threshold the basal synthesis of $\mathrm{BglG}$ is sufficient for antitermination and, as a result, $b g l G$ and the operon are expressed at high levels (Dole et al., 2002).

RpoS activity is controlled by multiple signals and at various levels. Predominant is the post-transcriptional control, for example its translation is regulated by the RNA-binding protein $\mathrm{Hfq}$ and small regulatory RNAs and its proteolysis is controlled by the response regulator RssB and the ClpXP protease (Hengge-Aronis, 2000). In addition, RpoS activity is affected by Crl, a $15 \mathrm{kDa}$ protein that is conserved among the Enterobacteriaceae (for example, it is present in the complete genome sequences of Salmonella enterica, Salmonella typhimurium LT2, Yersinia pestis and Vibrio cholerae) but 


\section{Table 1. E. coli $\mathrm{K}-12$ strains}

The relevant genotype of the constructed CSH50 derivatives refers to the $b g l$ and $r p o S$ loci, and the gpt-lac region. Mutations causing activation of the silent $b g l$ operon include $b g l$-CRP (a C to T exchange in the CRP binding site at position -66 relative to the transcription start), $b g l::$ IS1-R1243 (integration of IS1 in orientation II generating a target site duplication from -88 to -80 ), $b g l::$ IS5-H3 (an integration of IS5 in orientation II generating a target site duplication from position -92 to -89 ) and $b g l-42$ (a deletion of the upstream silencer, extending from position -77). Terminator mutation $t 1-L$ carries a three base exchange in the left stem of the inverted repeat structure (Dole et al., 2002). bgl $l^{0}$ indicates a silent wild type $b g l$ operon.

\begin{tabular}{|c|c|c|}
\hline Strain & Relevant genotype or structure & Construction $* /$ reference \\
\hline CSH50 & bgl $l^{0} \Delta($ lac-pro $)$ ara thi $(=\mathrm{S} 49)$ & Miller (1972) \\
\hline R1243 & CSH50 bgl::IS1-R1243 $\left(\mathrm{Bgl}^{+}\right)(=\mathrm{S} 157)$ & Schnetz \& Rak (1992) \\
\hline RH90 & MC4100 bgl rpoS359:: $\operatorname{Tn} 10(=\mathrm{S} 810)$ & Lange \& Hengge-Aronis (1991) \\
\hline S162 & CSH50 $\Delta b g l$-AC11 & Caramel \& Schnetz (1998) \\
\hline S308 & CSH50 attB: : [SpecR lacOP lacZ] & $\times$ pKESD13 \\
\hline S432 & CSH50 bgl-CRP & Spontaneous $\mathrm{Bgl}^{+}$mutant \\
\hline S484 & CSH50 bgl $l^{0} \Delta(\operatorname{argF}-l a c) \mathrm{U} 169 \mathrm{Pro}^{+}$ & Dole et al. (2002) \\
\hline S524 & CSH50 bgl $l^{0} \Delta l a c Z-Y 217[g p t-l a c]^{+}$ & Dole et al. (2002) \\
\hline S541 & S524 $\Delta b g l$-AC11 & Dole et al. (2002) \\
\hline S544 & $\mathrm{S} 524$ bgl-CRP & Dole et al. (2002) \\
\hline S887 & S541_rpoS359::Tn10 & Dole et al. (2002) \\
\hline S1071 & S524 rpoS359:: Tn10 & Dole et al. (2002) \\
\hline S1079 & S544 rpoS359:: $\operatorname{Tn} 10$ & Dole et al. (2002) \\
\hline S1142 & S541 attB::[SpecR WT $\mathrm{P}_{b g l} t 1-L$ bglG lacZ] & $\times$ pKESK11; Dole et al. (2002) \\
\hline S1144 & S541 attB: :[SpecR bgl-CRP $\mathrm{P}_{b g l}$ t1-L bglG lacZ] & $\times$ pKESK12; Dole et al. (2002) \\
\hline S1146 & S541 attB:: [SpecR bgl-42 $\mathrm{P}_{b g l}$ t1-L bglG lacZ] & $\times$ pKESK13; Dole et al. (2002) \\
\hline S1148 & S541 attB: :[SpecR bgl::IS5-H3 $\mathrm{P}_{b g l}$ t1-L bglG lacZ] & $\times$ pKESK14; Dole et al. (2002) \\
\hline S1155 & S1142 rpoS359:: Tn10 & Dole et al. (2002) \\
\hline S1157 & S1144 rpoS359:: Tn10 & Dole et al. (2002) \\
\hline S1159 & S1146 rpoS359:: Tn10 & Dole et al. (2002) \\
\hline S1161 & S1148 rpoS359:: Tn10 & Dole et al. (2002) \\
\hline S1163 & S162 attB: :[SpecR WT $\mathrm{P}_{b g l} t 1-L$ bglG lacZ] & $\times$ pKESK11 \\
\hline S1165 & $\mathrm{S} 162$ attB: :[SpecR bgl-CRP $\mathrm{P}_{\mathrm{bg} 1}$ t1-L bglG lacZ] & $\times$ pKESK 12 \\
\hline S1167 & S162 attB: : [SpecR bgl-42 $\mathrm{P}_{b g l}$ t1-L bglG lacZ] & $\times$ pKESK13 \\
\hline S1169 & S162 attB:: [SpecR bgl::IS5-H3 $\mathrm{P}_{b g l} t 1-L$ bglG lacZ] & $\times$ pKESK14 \\
\hline S1171 & S1163 rpoS359::Tn10 & $\times$ T4GT7(RH90) \\
\hline S1173 & S1165 rpoS359:: Tn10 & $\times \mathrm{T} 4 G T 7(\mathrm{RH} 90)$ \\
\hline S1177 & S1169 rpoS359:: Tn10 & $\times \mathrm{T} 4 G T 7(\mathrm{RH} 90)$ \\
\hline S1245 & S524 bgl::IS1-R1243 $\left[\mathrm{Bgl}^{+}\right]$ & Dole et al. (2002) \\
\hline S1250 & S1245 rpoS359:: Tn10 & Dole et al. (2002) \\
\hline S1274 & S541 attB::[SpecR lacOP lacZ] & $\times$ pKESD13 \\
\hline S1276 & S887 attB::[SpecR lacOP lacZ] & $\times$ pKESD13 \\
\hline S1305 & S308 rpoS359:: Tn10 & $\times \mathrm{T} 4 G T 7(\mathrm{RH} 90)$ \\
\hline S1484 & CSH50 rpoS359:: $\operatorname{Tn} 10$ & $\times \mathrm{T} 4 G T 7(\mathrm{RH} 90)$ \\
\hline S1486 & S162 rpoS359:: Tn10 & $\times \mathrm{T} 4 G T 7(\mathrm{RH} 90)$ \\
\hline S1489 & S484 rpoS359:: Tn10 & $\times \mathrm{T} 4 G T 7(\mathrm{RH} 90)$ \\
\hline S1491 & $\mathrm{S} 432 \Delta(\arg F-l a c) \mathrm{U} 169 \mathrm{Pro}^{+}$ & $\times \mathrm{T} 4 G T 7(\mathrm{MC} 4100)$ \\
\hline S1493 & R1243 $\Delta(\arg F-l a c)$ U169 Pro $^{+}$ & $\times \mathrm{T} 4 G T 7(\mathrm{MC} 4100)$ \\
\hline S1495 & R1243 rpoS359:: Tn10 & $\times \mathrm{T} 4 G T 7(\mathrm{RH} 90)$ \\
\hline S1497 & S432 rpoS359:: Tn10 & $\times \mathrm{T} 4 G T 7(\mathrm{RH} 90)$ \\
\hline S1499 & S1491 rpoS359:: Tn10 & $\times \mathrm{T} 4 G T 7(\mathrm{RH} 90)$ \\
\hline S1501 & S1493 rpoS359:: $\operatorname{Tn} 10$ & $\times \mathrm{T} 4 G T 7(\mathrm{RH} 90)$ \\
\hline S1503 & S541 attB:: [SpecR bgl::IS1-R1243 $\mathrm{P}_{b g l}$ t1-L bglG lacZ] & $\times$ pKESK 20 \\
\hline S1505 & S162 attB:: [SpecR bgl::IS1-R1243 $\mathrm{P}_{b q l}$ t1-L bglG lacZ] & $\times$ pKESK 20 \\
\hline S1507 & S887 attB::[SpecR bgl::IS1-R1243 $\mathrm{P}_{b g l} t 1-L$ bglG lacZ] & $\times$ pKESK 20 \\
\hline S1508 & S1486 attB:: [SpecR bgl::IS1-R1243 $\mathrm{P}_{b g l} t 1-L$ bglG lacZ] & $\times$ pKESK 20 \\
\hline S1512 & S1167 rpoS359::Tn10 & $\times \mathrm{T} 4 G T 7(\mathrm{RH} 90)$ \\
\hline
\end{tabular}

$* \times$ indicates that transductions and integrations into att $B$ were performed as described in Methods. 
shares no homology with other proteins. Crl was discovered because it is required for the RpoS-dependent expression of the $\operatorname{csg} B A$ operon encoding the fibronectin-binding curli fimbria (Crl = curli) (Olsen et al., 1989; Arnquist et al., 1992). Genetic analyses revealed that Crl stimulates to various extents all RpoS-dependent transcription processes and that $\mathrm{Crl}$ acts either in concert with RpoS or upstream of it in the same pathway (Pratt \& Silhavy, 1998).

Here the expression level of activated $b g l$ operon derivatives and $b g l-l a c Z$ reporter constructs were tested in strain CSH50 which carries a large deletion in the lac region, $\Delta(g p t-l a c)$, and derivatives that carry the smaller $\Delta(\arg F-l a c)$ deletion or the wild-type gpt-pro-lac $(\Delta l a c Z)$ region. In strain CSH50, activated bgl operon alleles are expressed at high levels. However, their expression is low in the other two deletion derivatives. In these strains the expression is increased up to 50-fold when a rpoS mutation is introduced (see also Dole et al., 2002). RpoS has no effect in CSH50. A complementation experiment shows that Crl, which is encoded in the $g p t-\arg F$ region, is required for the RpoS-dependent repression of the $b g l$ operon and responsible for these strain-dependent variations in the expression level of the $b g l$ operon.

\section{METHODS}

Strains and plasmids. Plates and liquid media were used as described by Dole et al. (2002). The genotypes of the E. coli K-12 strains used in this study are listed in Table 1. All strains are derivatives of CSH50 (Miller, 1972). [The $\Delta$ (pro-lac) X111 deletion present in CSH strains was mapped and renamed as $\Delta(g p t-l a c)$, Genetic Stock Center, http:// cgsc.biology.yale.edu/] Transductions were performed with phage T4GT7 (Wilson et al., 1979). For transduction of rpoS359::Tn10, cells were selected on LB tetracycline $\left(12 \mu \mathrm{g} \mathrm{ml}^{-1}\right)$ plates and for transduction of $\Delta(\operatorname{argF}-l a c)\left(\mathrm{Pro}^{+}\right)$ cells, minimal glucose B1 plates were used. Integrations of $b g l-l a c Z$ reporter constructs into the chromosomal phage lambda attachment site attB using plasmids pKESK11, pKESK12, pKESK13, pKESK14, pKESK20 or pKESD13 were performed and confirmed by PCR as described (Diederich et al., 1992; Dole et al., 2002). Plasmids were constructed according to standard techniques (Sambrook et al., 1989). The structure of plasmids pKESD13 and pKESD20 is similar to pKESK11 (Dole et al., 2002). Plasmid pKESD13 carries the lac operator promoter and the lacZ gene cloned into a pACYC backbone, including the kanamycin-resistance neo gene, attP, and the spectinomycin-resistance aadA gene. Plasmid pKESK 20 carries the $b g l$ promoter activated by integration of IS1 (allele $b g l-R 1243$ ) (Schnetz \& Rak, 1992), bgl terminator mutant $t 1-L, b g l G$ and $l a c Z$. Plasmid pKESK19 carries the $\mathrm{crl}$ gene cloned under control of the constitutive antisense promoter of the tetracycline resistance gene (Stüber \& Bujard, 1981). For its construction the $\mathrm{crl}$ gene fragment was amplified by PCR and cloned into pBR322, replacing the bla gene. In addition, a kanamycin resistance gene cassette was inserted into the tet gene. PCR fragments were sequenced after cloning. Details of constructions and compiled sequences of the plasmids are available upon request.

Phospho- $\boldsymbol{\beta}$-glucosidase and $\boldsymbol{\beta}$-galactosidase assays. These were performed as described by Miller (1972), Schnetz \& Rak (1988) and Dole et al. (2002). Cells were grown in M9 medium containing $1 \%$ glycerol and $0.66 \%$ Casamino acids (Difco). For induction of the $b g l$ operon, $0.5 \%(\mathrm{w} / \mathrm{v})$ salicin was added in the phospho- $\beta$-glucosidase assays. Cultures were inoculated from non-induced fresh overnight cultures grown in the same medium and cells were harvested after approximately $3 \mathrm{~h}$ growth at $37{ }^{\circ} \mathrm{C}$ at $\mathrm{OD}_{600}$ of $0 \cdot 5$. Enzyme activities were determined at least three times from at least two independent transformants or integration derivatives. Standard deviations were less than $10 \%$ in case of the $\beta$-galactosidase assays and less than $20 \%$ in case of the phospho- $\beta$-glucosidase assays.

\section{RESULTS AND DISCUSSION}

\section{Expression levels of activated $b g /$ operon derivatives are affected by deletions in the lac operon region}

The expression levels of the wild-type and activated $b g l$ operon alleles were tested in a set of isogenic CSH50 derivatives including a $\Delta(g p t-l a c)$, a $\Delta(\arg F-l a c)$ and a $\Delta l a c Z$ deletion (Fig. 1). The activated alleles of the $b g l$ operon that were tested include a mutant carrying an improved CRP-binding site or an integration of IS1 (Fig. $2)$. The expression level of the $b g l$ operon was determined in phospho- $\beta$-glucosidase assays from exponential-phase cultures grown in minimal M9 glycerol medium containing salicin as inducer. Phospho- $\beta$-glucosidase is encoded by the third gene of the $b g l$ operon (bglB).

As expected, the expression level of the silent wild-type bgl operon was low in all strains tested (Fig. 2a). However, the expression level of activated alleles varied significantly with the CSH50 strain and its deletion derivatives. These alleles are expressed at low levels in the $\Delta l a c Z$ and $\Delta(\operatorname{argF-lac})$ strains (Fig. 2). However, when the isogenic $\Delta(g p t-l a c)$ strain was used, the activated alleles were expressed at high levels. Expression of the IS1-activated allele is higher than that of the allele carrying an improved CRP-binding site. Low expression of activated alleles in the $\Delta l a c Z$ strain when cells are grown in medium containing glycerol was observed previously (Dole et al., 2002). Glycerol causes some catabolite repression of the operon (Lopilato \& Wright, 1990). Irrespective of the background, all strains carrying an activated operon show a Bgl-positive phenotype on salicin indicator plates. In the $\Delta l a c Z$ and $\Delta(\arg F-l a c)$ strain backgrounds, the introduction of a mutation in rpoS caused an up to 50-fold increase in the expression of the activated alleles (Fig. 2) (see also Dole et al., 2002), while RpoS had no further effect in the $\Delta(g p t-l a c)$ strain (Fig. 2). This indicated that a gene in the $g p t-\operatorname{argF}$ region is required for repression of the $b g l$ operon by RpoS (Fig. 1).

\section{Crl is required for the RpoS-mediated repression of the bgl operon}

The $c r l$ gene maps in the $g p t-\arg F$ region and thus was an obvious candidate for the observed difference. To test whether Crl complements the $\Delta(g p t-l a c)$ strain, plasmid pKESK19 carrying the $\mathrm{crl}$ gene under control of the constitutive antisense promoter of the tetracycline resistance gene (Stüber \& Bujard, 1981) was constructed. 


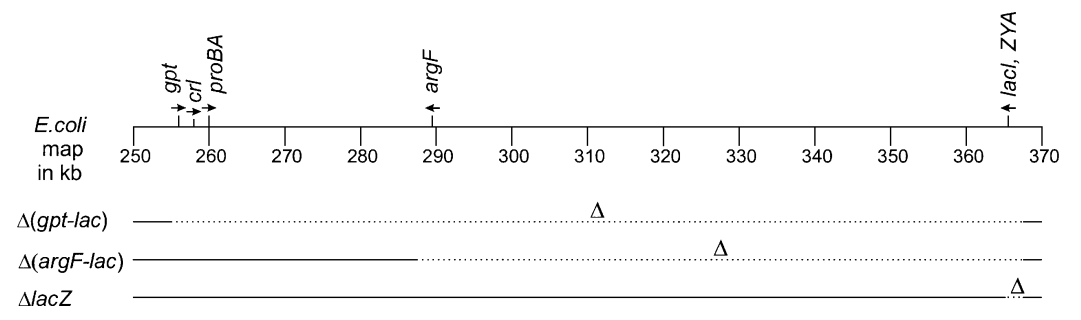

Fig. 1. Chromosomal map of the gpt-prolac operon region. Expression of the bgl operon was tested in strain background CSH50 (Miller, 1972) carrying a large deletion in the lac operon region $\Delta$ (gpt-lac), and CSH50-derived strains that carry a smaller deletion in the lac region, $\Delta(\arg F-l a c)$, or a deletion of the lacZ gene only (Dole et al., 2002). Map positions are given in kbp (gpt is at $256 \mathrm{kbp}, \mathrm{crl}$ is at $257.8 \mathrm{kbp}$, proBA is at $260.0 \mathrm{kbp}, \arg F$ is at 289.5 and lac is at $365 \cdot 5 \mathrm{kbp})$.

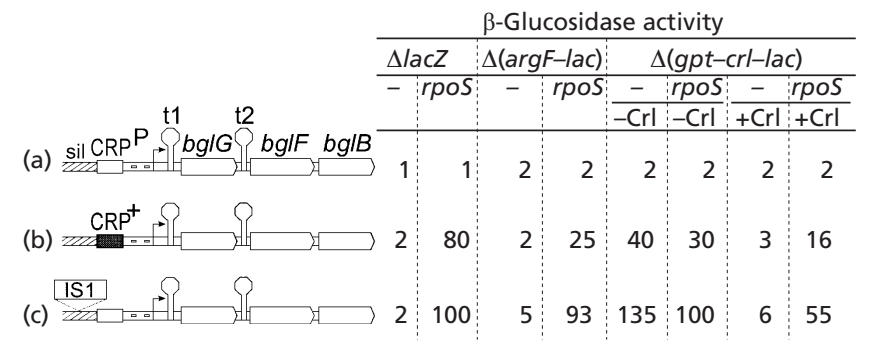

Fig. 2. RpoS and $\mathrm{Crl}$ are mutually required for repression of activated $b g l$ operon alleles. Expression levels of (a) the wildtype $b g /$ operon and alleles activated by (b) improvement of the CRP-binding site (CRP ${ }^{+}$, a $\mathrm{C}$ to $\mathrm{T}$ exchange at position -66 relative to the transcription start site), and (c) integration of IS1 (bgl::IS1-R1243) (Dole et al., 2002) are given in units of phospho$\beta$-glucosidase activity. Phospho- $\beta$-glucosidase is encoded by $\mathrm{BglB}$. BglF encodes the $\beta$-glucoside specific permease enzyme II ${ }^{\mathrm{Bgl}}$. Stem-loop structures $t 1$ and $t 2$ are rho-independent terminators at which BglG mediates antitermination. Expression levels were determined in $\mathrm{CSH} 50$ and derivatives, which differ in the size of the deletion in the lac operon region [ $\Delta$ (gpt-lac), $\Delta$ (argF-lac) and $\triangle / a c Z$ ]. Also tested were rpoS359::Tn10 (Lange \& HenggeAronis, 1991) mutants of CSH50 and its derivatives (Table 1). $+\mathrm{Crl}$ indicates expression levels of transformants of the $\mathrm{crl}$ expressing plasmid pKESK19. Strains used were: (a) S524, S1071, S484, S1489, CSH50 and S1484; (b) S544, S1079, S1491, S1499, S432 and S1497; (c) S1245, S1250, S1493, S1501, R1243 and S1495.

In transformants of the $\Delta(g p t-l a c)$ strain the repression of the activated $b g l$ operon alleles by RpoS was restored (Fig. 2; + Crl). As a control, in the RpoS-negative $\Delta(g p t-l a c)$ strain providing Crl in trans caused a less than twofold decrease [Fig. 2; $\Delta(g p t-c r l-l a c) r p o S$ $+\mathrm{Crl}]$. This demonstrates that $\mathrm{Crl}$ is required for the RpoS-mediated repression.

\section{$\mathrm{Crl}$ is required for the RpoS-mediated repression of bgl-lacZ reporter constructs}

Next a chromosomal $b g l-l a c Z$ reporter system was used in which expression is independent of the $\beta$-glucoside specific regulation by $\mathrm{BglG}$-mediated antitermination due to a mutation ( $t 1$-L) of terminator $t 1$ (Fig. 3) (Dole et al., 2002). In this system the changes in the transcription rate are not amplified by BglG-mediated antitermination and thus correlate with changes in the levels of expressed $\beta$-galactosidase (see Introduction). RpoS has an approximately threefold effect on the expression level of the $b g l-l a c Z$ reporter gene system

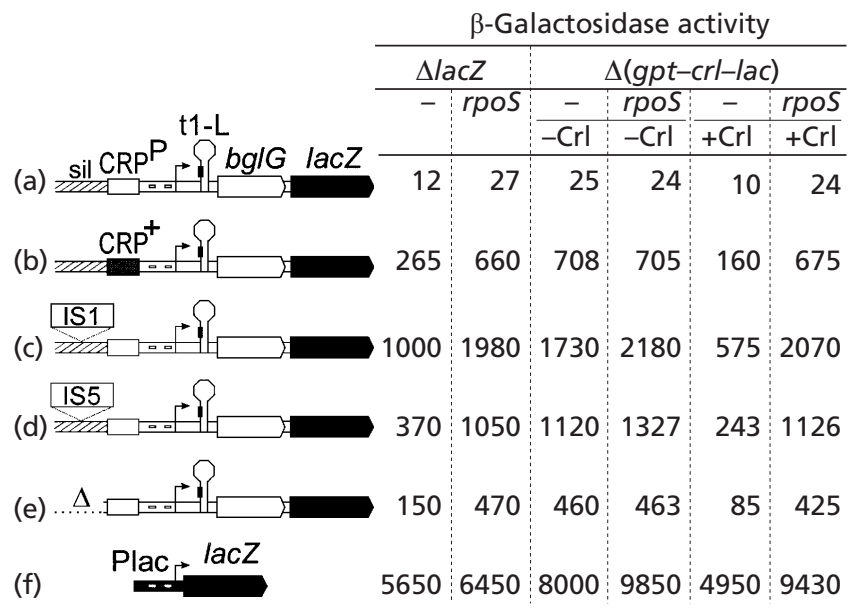

Fig. 3. $\mathrm{Crl}$ is required for the RpoS-mediated repression of BglG-independent $b g l-l a c Z$ reporter constructs. Chromosomally encoded bgl-lacZ reporter constructs (Dole et al., 2002) carrying the wild-type bgl promoter (a) or alleles activated by (b) improvement of the CRP-binding site $\left(\mathrm{CRP}^{+}\right)$, (c) integration of IS1, (d) integration of IS5 and (e) a deletion of the upstream silencer ( $\Delta 2$ in Table 1) were tested in the CSH50 $\Delta / a c Z$ derivative and in $\mathrm{CSH} 50, \Delta$ (gpt-crl-lac) $\left[\mathrm{rpoS}^{+}\right]$, and the rpoS359::Tn10 mutant derivatives of these strains. $\mathrm{Crl}$ was provided in trans $(+\mathrm{Crl})$ using plasmid pKESK19. All the bgl-lac $Z$ constructs carry mutation $t 1-L$ of the first terminator, a $3 \mathrm{bp}$ exchange in the left stem of the inverted repeat forming the terminator, which renders expression independent of BglGmediated antitermination. As a control ( $f$ ) expression of a lacOP-lacZ construct integrated into attB (Diederich et al., 1992; Dole et al., 2002) was also tested. Strains used were: (a)

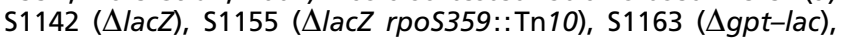
and S1171 ( $\Delta$ (gpt-lac) rpoS359::Tn10); (b) S1144, S1157, S1165 and S1173; (c) S1503, S1507, S1505 and S1508; (d) S1146, S1159, S1167 and S1512; and (e) S1274, S1276, S308 and S1305.

(Dole et al., 2002). Analysed were bgl-lacZ fusions that carry the wild-type $b g l$ promoter (Fig. 3a) or activated alleles, including the mutants that carry the improved CRP-binding site (Fig. 3b) or the integration of IS1 (Fig. 3c). In addition, mutants carrying an integration of IS5 (Fig. 3d) and a deletion of the upstream silencer were tested (Fig. 3e) (Dole et al., 2002). Expression levels are given in units of $\beta$-galactosidase activity and thus the values are higher than in the phospho- $\beta$-glucosidase assay shown in Fig. 2. Similar to the results obtained using the $b g l$ operon, the $b g l-l a c Z$ constructs were repressed by RpoS when $\mathrm{Crl}$ was present $(\Delta l a c Z)$ but not when Crl was missing ( $\Delta g p t-c r l-l a c)$ (Fig. 3). Repression 
of the activated alleles by RpoS is approximately two- to threefold (Fig. 3). Again, providing Crl in trans restored repression by RpoS in the $\Delta g p t-c r l-l a c$ strain, but had no effect in the rpoS mutant of this strain (Fig. 3). As a control, the expression of a lacOP-lacZ fragment integrated into att $B$ varied only moderately in the various strain backgrounds (Fig. 3).

\section{Role of RpoS and $\mathrm{Crl}$ in bgl operon regulation}

The $b g l$ operon is regulated by various pleiotropic regulators including H-NS and CRP, as essential factors. In addition, rather moderate changes (approximately two- to threefold) in the transcription rate of the operon can be amplified post-transcriptionally into large changes in the expression level (Dole et al., 2002). Therefore, the expression level of the $b g l$ operon and the effect of any non-essential factor (for example RpoS and Crl) involved in $b g l$ operon regulation may vary significantly depending on the strain background and the growth conditions that are used. RpoS causes a moderate repression of the $b g l$ promoter of activated $b g l$ operon derivatives (Dole et al., 2002). The finding that the RpoS-mediated repression requires Crl supports earlier findings that Crl stimulates RpoS activity. How Crl stimulates RpoS is unknown. Interestingly, RpoS is required for silencing of a $b g l-l a c Z$ fusion by a truncated $\mathrm{H}-\mathrm{NS}$ protein in a $\Delta(g p t-l a c)$, i.e. Crl-negative, strain (Ohta et al., 1999). Likewise, the contribution of Crl to the RpoS-mediated transcription of the Curli $(\operatorname{csg} B A)$ operon varies with the strain background (Olsen et al., 1989; Provence \& Curtiss, 1992). This indicates that the enhancement of RpoS-mediated regulation by $\mathrm{Crl}$ may depend on H-NS and other pleiotropic regulators.

\section{ACKNOWLEDGEMENTS}

I thank Sandra Kühn for excellent technical assistance, and rotation students Bettina Pies and Monika Przybytek for contribution to the experiments. The work was funded by the Deutsche Forschungsgemeinschaft through SFB274.

\section{REFERENCES}

Arnquist, A., Olsén, A., Pfeifer, J., Russell, D. G. \& Normark, S. (1992). The Crl protein activates cryptic genes for curli formation and fibronectin binding in Escherichia coli HB101. Mol Microbiol 6, 2443-2452.

Caramel, A. \& Schnetz, K. (1998). Lac and Lambda repressor relieve silencing of the Escherichia coli bgl promoter: activation by alteration of a repressing nucleoprotein complex. J Mol Biol 284, 875-883.

Caramel, A. \& Schnetz, K. (2000). Antagonistic control of the E. coli bgl promoter by FIS and CAP in vitro. Mol Microbiol 36, 85-92.

Defez, R. \& de Felice, M. (1981). Cryptic operon for $\beta$-glucoside metabolism in Escherichia coli K12: genetic evidence for a regulatory protein. Genetics $\mathbf{9 7}, 11-25$.

Diederich, L., Rasmussen, L. J. \& Messer, W. (1992). New cloning vectors for integration into the lambda attachment site attB of the Escherichia coli chromosome. Plasmid 28, 14-24.

Dole, S., Kühn, S. \& Schnetz, K. (2002). Post-transcriptional enhancement of Escherichia coli bgl operon silencing by limitation of BglG-mediated antitermination at low transcription rates. Mol Microbiol 43, 217-226.

Free, A., Williams, R. M. \& Dorman, C. J. (1998). The StpA protein functions as a molecular adapter to mediate repression of the $b g l$ operon by truncated H-NS in Escherichia coli. J Bacteriol 180, 994-997.

Free, A., Porter, M. E., Deighan, P. \& Dorman, C. J. (2001). Requirement for the molecular adapter function of StpA at the Escherichia coli $\mathrm{bgl}$ promoter depends upon the level of truncated H-NS protein. Mol Microbiol 42, 903-918.

Giel, M., Desnoyer, M. \& Lopilato, J. (1996). A mutation in a new gene, bglJ, activates the $b g l$ operon in Escherichia coli K-12. Genetics 143, 627-635.

Hengge-Aronis, R. (2000). The general stress response in Escherichia coli. In Bacterial Stress Responses, pp. 161-178. Edited by G. Storz and R. Hengge-Aronis. Washington, DC: American Society for Microbiology.

Higgins, C. F., Dorman, C. J., Stirling, D. A., Waddell, L., Booth, I. R., May, G. \& Bremer, E. (1988). A physiological role for DNA supercoiling in the osmotic regulation of gene expression in $S$. typhimurium and E. coli. Cell 52, 569-584.

Lange, R. \& Hengge-Aronis, R. (1991). Identification of a central regulator of stationary-phase gene expression in Escherichia coli. Mol Microbiol 5, 49-59.

Lopilato, J. \& Wright, A. (1990). Mechanisms of activation of the cryptic bgl operon of Escherichia coli K-12. In The Bacterial Chromosome, pp. 435-444. Edited by K. Drlica and M. Riley. Washington, DC: American Society for Microbiology.

Miller, J. H. (1972). Experiments in Molecular Genetics. Cold Spring Harbor, New York: Cold Spring Harbor Laboratory.

Ohta, T., Ueguchi, C. \& Mizuno, T. (1999). rpoS function is essential for $b g l$ silencing caused by C-terminally truncated H-NS in Escherichia coli. J Bacteriol 181, 6278-6283.

Olsen, A., Jonsson, A. \& Normark, S. (1989). Fibronectin binding mediated by a novel class of surface organelles on Escherichia coli. Nature 338, 652-655.

Pratt, L. A. \& Silhavy, T. J. (1998). Crl stimulates RpoS activity during stationary phase. Mol Microbiol 29, 1225-1236.

Provence, D. L. \& Curtiss, R., III (1992). Role of $\mathrm{crl}$ in avian pathogenic Escherichia coli: a knockout mutation of $\mathrm{crl}$ does not affect hemagglutination activity, fibronectin binding, or Curli production. Infect Immun 60, 4460-4467.

Reynolds, A. E., Felton, J. \& Wright, A. (1981). Insertion of DNA activates the cryptic $b g l$ operon of E. coli K12. Nature 293, 625-629.

Reynolds, A. E., Mahadevan, S., LeGrice, S. F. J. \& Wright, A. (1986). Enhancement of bacterial gene expression by insertion elements or by mutation in a CAP-cAMP binding site. J Mol Biol 191, 85-95.

Sambrook, J., Fritsch, E. F. \& Maniatis, T. (1989). Molecular Cloning: a Laboratory Manual, 2nd edn. Cold Spring Harbor, NY: Cold Spring Harbor Laboratory.

Schaefler, S. \& Maas, W. K. (1967). Inducible system for the utilization of $\beta$-glucosides in Escherichia coli. II. Description of mutant types and genetic analysis. J Bacteriol 93, 264-272.

Schnetz, K. (1995). Silencing of Escherichia coli bgl promoter by flanking sequence elements. EMBO J 14, 2545-2550.

Schnetz, K. \& Rak, B. (1988). Regulation of the $b g l$ operon of Escherichia coli by transcriptional antitermination. EMBO J 7, 3271-3277.

Schnetz, K. \& Rak, B. (1992). IS5: a mobile enhancer of 
transcription in Escherichia coli. Proc Natl Acad Sci US A 89, 1244-1248.

Singh, J., Mukerji, M. \& Mahadevan, S. (1995). Transcriptional activation of the $b g l$ operon of $E$. coli: negative regulation by DNA structural elements near the promoter. Mol Microbiol 17, 1085-1092.

Stüber, D. \& Bujard, H. (1981). Organization of transcriptional signals in plasmids pBR322 and pACYC184. Proc Natl Acad Sci US A 78, 167-171.

Tsui, H.-C. T., Leung, H.-C. E. \& Winkler, M. E. (1994). Characterization of broadly pleiotropic phenotypes caused by an $h f q$ insertion mutation in Escherichia coli K-12. Mol Microbiol 13, $35-49$.

Ueguchi, C., Ohta, T., Seto, C., Suzuki, T. \& Mizuno, T. (1998). The leu $\mathrm{O}$ gene product has a latent ability to relieve the $b g l$ silencing in Escherichia coli. J Bacteriol 180, 190-193.

Wilson, G. G., Young, K. Y. K., Edlin, G. J. \& Konigsberg, W. (1979). High-frequency generalised transduction by bacteriophage T4. Nature 280, 80-82.

Received 25 March 2002; revised 3 May 2002; accepted 7 May 2002. 